\title{
Key-Recovery Attack on the ASASA Cryptosystem With Expanding S-Boxes
}

\author{
Henri Gilbert, Jérôme Plût, and Joana Treger \\ ANSSI, France ${ }^{\star}$ \\ \{henri.gilbert,jerome.plut,joana.marim\}@ssi.gouv.fr
}

\begin{abstract}
We present a cryptanalysis of the ASASA public key cipher introduced at ASIACRYPT 2014 [3]. This scheme alternates three layers of affine transformations A with two layers of quadratic substitutions S. We show that the partial derivatives of the public key polynomials contain information about the intermediate layer. This enables us to present a very simple distinguisher between an ASASA public key and random polynomials. We then expand upon the ideas of the distinguisher to achieve a full secret key recovery. This method uses only linear algebra and has a complexity dominated by the cost of computing the kernels of $2^{26}$ small matrices with entries in $\mathbb{F}_{16}$.
\end{abstract}

\section{Introduction}

A long-standing challenge in asymmetric cryptography is to bring asymmetric cryptography closer to symmetric cryptography by designing public key schemes whose overall structure and elementary operations are similar to those used in mainstream block ciphers such as AES. Solving this appealing but difficult challenge would not only increase the diversity in asymmetric cryptography, but might also help reducing the considerable performance gap between asymmetric cryptography and symmetric cryptography (the latter currently being more efficient by several orders of magnitude). This might as well allow the emergence of symmetric algorithms with some extra features, as for instance symmetric encryption schemes with a secure white-box implementation. Until 2014 however, as far as we know, all attempts of public key scheme designs with block cipher features, e.g. $[10,16,15]$, eventually turned out to be weak $[13,18,1,8,7]$.

Asymmetric ASASA Schemes. Some new candidate solutions to the above challenge were proposed in a paper published at AsIACRYPT 2014 by Biryukov et al. [3]. One conducting idea for the new designs stems from the observations

(C) IACR 2015. This article is the final version submitted by the author(s) to the IACR and to Springer-Verlag on June 5, 2015. The version published by SpringerVerlag is available at \$DOI.

* This work was partially supported by the French National Research Agency through the BRUTUS project (contract ANR-14-CE28-0015). 
that: (1) Traditional SPN block ciphers such as AES can be viewed as an alternance of (at least partly secret) affine transformations A and S-box layers S, and generally comprise a substantial number of A rounds (essentially 10 in the case of AES-128); as shown by Biryukov and Shamir [4], some efficient generic attacks exist for the SASAS structure with secret $\mathrm{S}$ and $\mathrm{A}$ layers and small bijective S-boxes. (2) The efforts to design public key schemes with an alternance of $A$ and $S$ layers mainly focused so far on multivariate schemes with an ASA structure, with one single large S-box described by low degree equations over a finite field. ${ }^{1}$ Based on the former considerations, the authors of [3] advocate for use of public multivariate schemes with an ASASA structure, i.e. with the simplest possible structure for which no generic attack is known in the case of small bijective S-boxes - or more generally of injective S-boxes whose non-zero linear combinations of outputs are not too strongly biased.

More precisely, the authors of [3] proposed a public-key encryption scheme named the asymmetric ASASA scheme with expanding S-boxes, conjecturing that it offers a comfortable security margin with respect to the potential lines of attack identified in their security analysis. This scheme uses small input-expanding injective quadratic S-boxes. Since the these S-boxes have a length expansion factor of 2 , the whole scheme has a length expansion factor of 4 . The standard plaintext and ciphertext length for this scheme are respectively 128 and 512 bits.

While this works focuses exclusively on the ASASA scheme with expanding S-boxes, the same authors also proposed in [3] a second public-key encryption scheme based on the ASASA structure, named the $\chi$-scheme. Indeed, this alternative construction makes use of Daemen's bijective quadratic S-box $\chi$ based on cellular automata [5] and also used in various recent hash functions. The standard plaintext and ciphertext length is 128 bits. In this $\chi$-scheme, one single large S-box is used at each S layer. In their security analysis though, the authors of [3] consider many attacks on weakened versions of the $\chi$-scheme and conclude that the security margin of the $\chi$-scheme must be lower than that of the expanding scheme. They therefore express some caveats on its security and only "offer it as a cryptanalytic challenge, but not for practical use", unlike the expanding scheme.

In both ASASA public key encryption schemes, quadratic S-boxes are being used. The public key consists of the quartic equations of the encryption function and the private key consists of the specification of the A and S layers and of some perturbation polynomials, which are added to a few components of the vector representing the output of the second $\mathrm{S}$ layer. ${ }^{2}$ Another property of these public

\footnotetext{
${ }^{1}$ While a noticeable exception is the multivariate scheme $R 2$ [14] that contains two S layers with small S-boxes, one weakness highlighted by attacks on $R 2$ or its variant $R 2^{-}$that were eventually discovered is the fact that the $R 2$ S-boxes are not injective.

${ }^{2}$ While the role of perturbations is essential in the case of the $\chi$-scheme since its variants without perturbations are reported in [3] to be vulnerable to efficient Gröbner basis attacks, in the case of the expanding scheme, perturbations are mostly introduced to provide some extra resistance against decomposition attacks that could potentially reduce the ASASA structure to the functional composition of two ASA structures.
} 
key encryption schemes is that they can also be viewed as symmetric ciphers with a decent encryption and decryption speed and the following extra feature: as an alternative to using the secret key to efficiently encrypt, the public key provides a slower strong white-box implementation of the encryption function - i.e. an obfuscated implementation that is conjectured to prevent that the decryption function be derivable by an adversary who has full access to it.

Our contribution. In this paper, we present an efficient attack on the ASASA scheme with expanding S-boxes. The starting point for our attack is the analysis of the homogeneous cubic part of the derivatives of the (quartic) polynomials of the public key. We first show that this analysis provides a distinguisher that allows to tell apart the public key of the scheme from a vector of random quartic polynomials. We then describe how to leverage the first information about the secret key provided by the distinguisher to retrieve the intermediate values that lie between the two S-layers, for an equivalent representation of the scheme. At this point, we are essentially left with the problem of solving two quadratic ASA layers. Though generic techniques to solve this problem exist, we give our own algorithm, that is well-adapted to the scheme considered. The overall complexity of the attack is equivalent to at most $2^{26}$ computations of kernels of matrices of size $64 \times 96$ over the finite field $\mathbb{F}_{16}$. We estimate the corresponding computational time to a few CPU-hours, which places this cryptanalysis well within practical limits.

This paper is organised as follows. Section 1 provides a description of the expanding ASASA scheme and presents some useful preliminary results. Section 2 introduces a distinguisher for this scheme that can be used to derive some first information on the secret key. Finally, Section 3 shows how to efficiently derive an equivalent secret key from the public key.

\section{The ASASA Cryptosystem}

\subsection{Definition and First Notations}

The two asymmetric ASASA schemes of [3] are composed of polynomial transformations over the base field $k=\mathbb{F}_{16}$; they are obtained by alternating three $k$-affine layers and two non-linear polynomial-based S layers. The ASASA scheme with expanding S-boxes, on which we are focusing, involves S-boxes whose output is twice as big as their input; 32 perturbation polynomials of degree four are also applied just before the last affine transform. More precisely, each S-box maps a 4 -tuple of $k$-values onto an 8-tuple of $k$-values, defined as degree 2 polynomials over $k$ in the inputs. The resulting scheme, which we simply call the ASASA cryptosystem in the remaining of this paper for simplification purposes, has then degree 4 over $k$. Going into details, the private key of the ASASA cryptosystem consists of:

- Three uniformly random invertible affine transformations $\mathcal{A}^{x}$ of $k^{32}$, $\mathcal{A}^{y}$ of $k^{64}$, and $\mathcal{A}^{z}$ of $k^{128}$; 


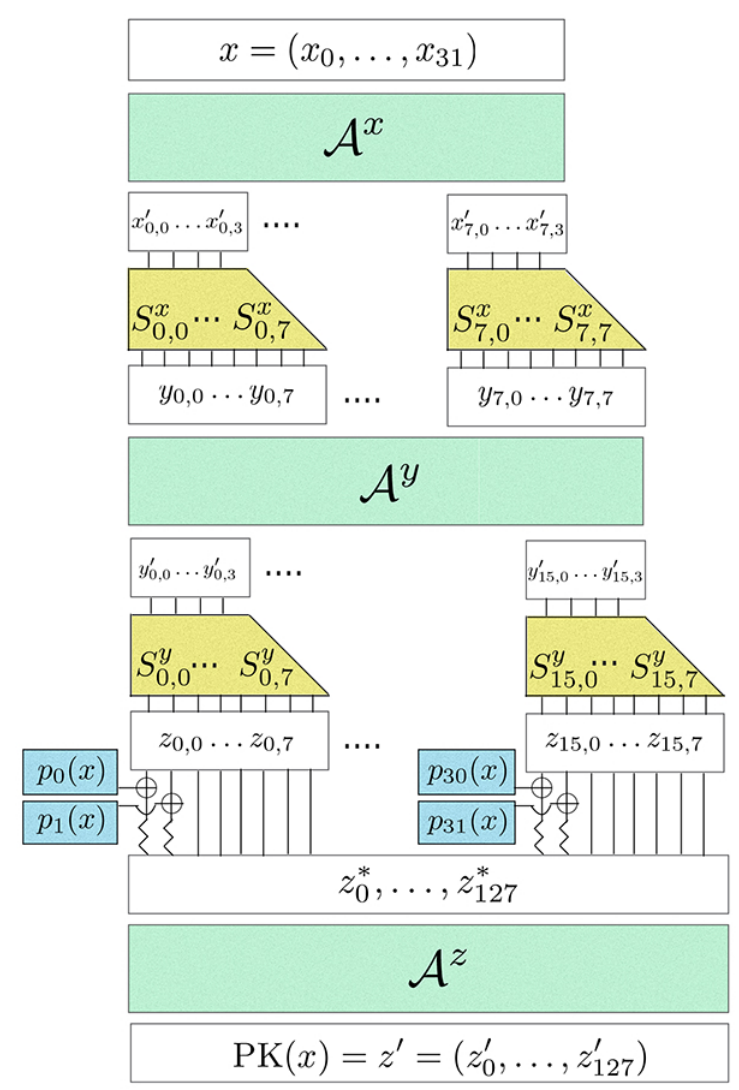

Fig. 1. The ASASA cryptosystem with expanding S-boxes.

- Two sets of uniformly random quadratic functions from $k^{4}$ to $k^{8}$ corresponding to the first and second S-box layer $S^{x}=$ $\left\{S_{0,0}^{x}, \ldots, S_{0,7}^{x}, \ldots S_{7,0}^{x}, \ldots S_{7,7}^{x}\right\}$, that determines 8 S-boxes $S_{0}^{x}, \ldots S_{7}^{x}$, and $S^{y}=\left\{S_{0,0}^{y}, \ldots, S_{7,0}^{y}, \ldots S_{15,0}^{y}, \ldots, S_{15,7}^{y}\right\}$, that determines 16 S-boxes $S_{0}^{y}, \ldots S_{15}^{y}$;

- Thirty-two uniformly random quartic perturbation polynomials $p_{0}, \ldots, p_{31}$ on $k^{32}$.

The public key and the associated public encryption function are derived from the secret key as illustrated at Figure 1, following those successive steps:

(i) The plaintext state is the tuple of variables $x=\left(x_{0}, \ldots, x_{31}\right) \in k^{32}$;

(ii) Let $x^{\prime}=\mathcal{A}^{x} \cdot x \in k^{32}$;

(iii) Define $y=\left(y_{0,0}, \ldots, y_{7,7}\right) \in k^{64}$ as $y_{r, i}=S_{r, i}^{x}\left(x_{r, 0}^{\prime}, \ldots, x_{r, 3}^{\prime}\right)$, for $r, i=0 \ldots 7$;

(iv) Let $y^{\prime}=\mathcal{A}^{y} \cdot y \in k^{64}$;

(v) Define $z=\left(z_{0,0}, \ldots, z_{15,7}\right) \in k^{128}$ as $z_{r, i}=S_{r, i}^{y}\left(y_{r, 0}^{\prime}, \ldots, y_{r, 3}^{\prime}\right)$, for $i=0 \ldots 7$ and $r=0 \ldots 15$; 
(vi) For $r \in\{0,15\}$, do $z_{8 r}^{\star} \leftarrow z_{r, 0}+\mathrm{p}_{2 r}(x), z_{8 r+1}^{\star} \leftarrow z_{r, 1}+\mathrm{p}_{2 r+1}(x)$, and $z_{8 r+i}^{\star} \leftarrow$ $z_{r, i}$ for $i=2 \ldots 7$ (the two first components out of 8 contiguous components of $z$ are modified);

(vii) The public key $\operatorname{PK}(x)$ is the vector of 128 polynomials over $k, z^{\prime}=\mathcal{A}^{z} \cdot z^{\star} \in$ $\left(k\left[x_{0}, \ldots x_{31}\right]\right)^{128}$; the public encryption function is the associated function from $k^{32}$ to $k^{128}$.

Dimension of the secret and public key spaces. Since the dimensions of various vector spaces are central in our analysis, we compute the size of the secret and public key spaces. We do not find the exact same numbers as the original authors $[3,2.5]$, although the order of magnitude is the same. An affine transform on $n$ variables is representable by a matrix of size $n \times(n+$ 1 ); therefore, the three A layers have a key size of $32 \times 33+64 \times 65+128 \times$ $129=21728$ elements of $\mathbb{F}_{16}$. An S-box output is an (inhomogeneous) quadratic polynomial in four variables, and is therefore described by $\left(\begin{array}{l}6 \\ 2\end{array}\right)=15$ coefficients. (The dimensions of spaces of homogeneous polynomials are as given below in section 1.3 of this paper; inhomogeneous polynomials in $n$ variables correspond bijectively to homogeneous polynomials of the same degree in $n+1$ variables). Therefore, the two $S$ layers have a key size of $24 \times 8 \times 15=2880$ elements of $\mathbb{F}_{16}$. In total, the secret key size is 24608 elements of $\mathbb{F}_{16}$, or approximately $2^{13.6}$ bytes of data. This does not, however, count the perturbation polynomials, which occupy a space of $32 \times\left(\begin{array}{c}36 \\ 4\end{array}\right)$ elements, or $2^{19.8}$ bytes of data. The public key is a set of $128 \times\left(\begin{array}{c}36 \\ 4\end{array}\right)$ elements of $\mathbb{F}_{16}$, or $2^{21.8}$ bytes of data.

\subsection{Equivalent Simple Keys}

As for most multivariate cryptosystems, there are multiple private keys that correspond to a given ASASA public key, and we show here that each secret key is equivalent to a simpler one, that we describe. We also redefine the ASASA system in terms of those "simple" keys, which make our attack easier to explain; we point out that this simplification is purely cosmetic though and that our attack does apply to the ASASA system as described in [3].

First, since for each $r=0, \ldots, 15$, the two outputs $z_{r, 0}, z_{r, 1}$ of the S-box $S_{r}^{y}$ are added to arbitrary perturbation polynomials $p_{i}$, we obtain the same public key when replacing $p_{2 r}, p_{2 r+1}$ by $p_{2 r}+z_{r, 0}, p_{2 r+1}+z_{r, 1}$ and $S_{r, 0}^{y}, S_{r, 1}^{y}$ by zero.

Let $\mathcal{A}^{x}=A^{x}+a^{x}$ and $\mathcal{A}^{y}=A^{y}+a^{y}$ be the decomposition of $\mathcal{A}^{x}$ and $\mathcal{A}^{y}$ as their linear part plus their constant. We can actually assume that $\mathcal{A}^{x}=$ $A^{x}$ and $\mathcal{A}^{y}=A^{y}$, as it is always possible to consider a modified $S^{y}$ S-box layer where the first addition by $a^{x}$ and the second addition by $a^{y}$ are absorbed by the polynomials of $S^{y}$. The same goes for $\mathcal{A}^{z}$, where the addition of the 32 components with index $8 r$ and $8 r+1$ of $a^{z}$ can be viewed as part of the perturbation polynomials, so that $S_{r, 0}^{y}$ and $S_{r, 1}^{y}$ are still zero. This shows that we can assume $a^{x}=a^{y}=a^{z}=0$ and consider from now on $A^{x}, A^{y}$ and $A^{z}$ instead of $\mathcal{A}^{x}, \mathcal{A}^{y}$ and $\mathcal{A}^{z}$. 


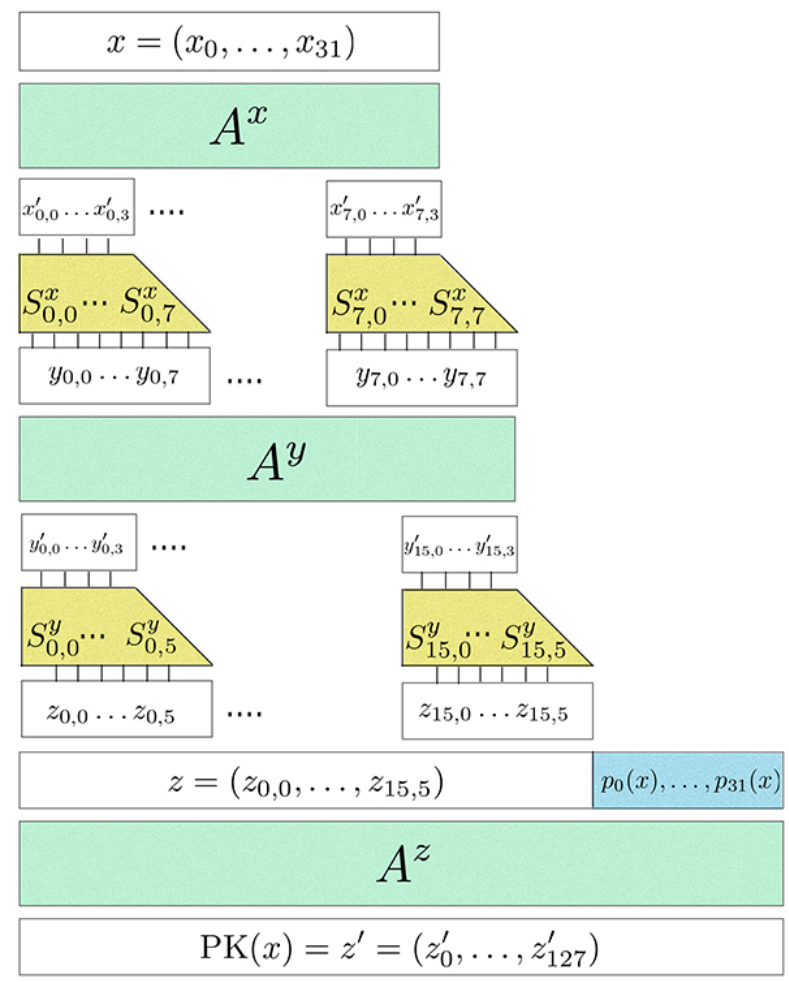

Fig. 2. The ASASA cryptosystem with expanding S-boxes, equivalent representation.

Finally, notice that it is always possible to compose the output of the quadratic map $S_{i}^{x}$ by a linear transform, and the corresponding input block of the linear map $A^{y}$ by its inverse. The same applies to $S_{i}^{y}$ and $A^{z}$, for $i \neq 8 r, 8 r+1$, i.e. as long as the corresponding 32 zero polynomials of $S^{y}$ are not affected.

To sum up, the description of the ASASA private key of Section 1.1 is equivalent to the following:

- Three uniformly random invertible linear transformations $A^{x}$ of $k^{32}$, $A^{y}$ of $k^{64}$ and $A^{z}$ of $k^{128}$;

- Two sets of $8 \times 8$ and $16 \times 6$ uniformly random quadratic functions on $k^{4}$, $S^{x}=\left\{S_{0,0}^{x}, \ldots, S_{7,7}^{x}\right\}$ and $S^{y}=\left\{S_{0,0}^{y}, \ldots, S_{15,5}^{y}\right\}$

- A set of 32 uniformly random quartic polynomials $p_{0}, \ldots, p_{31}$ on $k^{32}$.

In the remaining of this paper, we shall always consider such simple private keys; the corresponding encryption mechanism is illustrated at Figure 2.

\subsection{Notations and Preliminaries}

We write $k=\mathbb{F}_{16}$ for the finite field with 16 elements. Throughout this work, we let $q=|k|=16$. 
Homogeneous polynomials. We write $H_{d, n}$ for the space of homogeneous polynomials of degree $d$ over $n$ variables (over the base field $k=\mathbb{F}_{16}$ ); it is a vector space of dimension $\left(\begin{array}{c}n+d-1 \\ d\end{array}\right)$ [17, 2.2.1]. Throughout this paper, we shall usually work with the vector space $H_{d, 32}$ of homogeneous polynomials of degree $d$ on the 32 input variables $x_{i}$. We shall write $H_{d}$ instead of $H_{d, 32}$.

Let $f\left(x_{1}, \ldots, x_{n}\right)$ be a polynomial. For any integer $d$, we write $f_{(d)}$ for the degree $d$ homogeneous part of $f$.

Vector spaces. Given subspaces $E \subset H_{d, n}$ and $E^{\prime} \subset H_{d^{\prime}, n}$, we define $E \cdot E^{\prime}$ as the subspace of $H_{d+d^{\prime}, n}$ generated by all the products $u \cdot u^{\prime}$ for $u \in E$ and $u^{\prime} \in E^{\prime}$. We also define $D$ as the vector space generated by the 32 derivations $\partial_{i}=\partial / \partial x_{i}$. For any vector space $E \subset H_{d, n}$, we define $D E \subset H_{d-1, n}$ as the vector space generated by all $\delta(u)$ for $\delta \in D$ and $u \in E$.

Counting matrices of a given rank. We introduce the following notations, used in the computation of the number of matrices with given size and rank. For any integers $n \geq d$, we define

$$
[n, d]=\prod_{i=0}^{d-1} q^{n}-q^{i} \quad \text { and } \quad\left[\begin{array}{l}
n \\
d
\end{array}\right]=\frac{[n, d]}{[d, d]} .
$$

We omit the value $q$ from these notations, since we shall always use $q=16$. We use the following classical result (see [12, VII.19]).

Proposition 1. Let $m, n, d$ be three integers.

(i) There are exactly $[n, d]$ injective maps from $k^{d}$ to $k^{n}$.

(ii) There are exactly $\left[\begin{array}{l}n \\ d\end{array}\right]$ subspaces of dimension $d$ of $k^{n}$.

(iii) There are exactly $\frac{[n, d] \cdot[m, d]}{[d, d]}$ matrices of size $m \times n$ with rank exactly $d$.

Proposition 2. Let $V$ be a vector space of dimension d over $k=\mathbb{F}_{16}$. A set of $n$ uniformly random, independent vectors $v_{i} \in V$ generates $V$ with overwhelming probability if $n \geq d+32$.

Proof. Let $\pi(n, d)$ be the probability that a random matrix of size $n \times d$ has maximal rank $d$. We see by Proposition 1 that

$$
\pi(n, d)=\frac{[n, d]}{q^{n d}}=\prod_{i=0}^{d-1} 1-q^{-(n-i)} .
$$

For $n>d \gg 0$, since $q^{-n} \ll 1$, we have the asymptotic expansion

$$
\pi(n, d)=\exp \left(\sum_{i=0}^{d-1} \log \left(1-q^{-(n-i)}\right)\right) \simeq \exp \left(-q^{-n} \frac{q^{d}-1}{q-1}\right) .
$$


For any $\varepsilon>0$, we find that

$$
\pi(n, d)>1-\varepsilon \quad \text { iff } \quad n>\log _{q} \frac{q^{d}-1}{q-1}-\log _{q} \log \frac{1}{1-\varepsilon} .
$$

Using $\varepsilon=2^{-128}$ as our definition of "overwhelming probability", the above condition (4) becomes $n \geq d+32$.

Expected behaviour of derivatives and product of random polynomials. We shall use throughout this work the following heuristics about the derivatives and products of random polynomials.

For $f_{1}, \ldots, f_{r}$ uniformly random, independent elements of $H_{d, n}$ :

(i) if $r<\frac{1}{n} \operatorname{dim} H_{d-1, n}$, then the $n r$ derivatives $\partial_{i} f_{j}$ behave like uniformly random, independent elements of $H_{d-1, n}$;

(ii) if $r<\frac{1}{n} \operatorname{dim} H_{d+1, n}$, then the $n r$ products $x_{i} f_{j}$ behave like uniformly random, independent elements of $H_{d+1, n}$.

In particular, according to Proposition 2, we expect that, with overhelming probability, if $n r \leq \operatorname{dim} H_{d-1, n}-32$, then the $\partial_{i} f_{j}$ are free in $H_{d-1, n}$; if $n r \leq$ $\operatorname{dim} H_{d+1, n}-32$, then the $x_{i} f_{j}$ are free in $H_{d+1, n}$. Although giving a detailed proof of these facts would be out of the scope of this work, we obtained an empiric confirmation in the cases of interest to us (namely $n=32$, and either $d=4, r=128$ for derivation, or $d=2, r=64$ for multiplication), as well as of the bounds of validity.

\section{A Simple Distinguisher}

We shall see that the ASASA cryptosystem presents the same flaw as several multivariate cryptosystems, that is, it is possible to distinguish the equations of the public key for the ASASA scheme from random polynomials of the same degree over $k$. The distinguisher we present here is extremely simple: namely, computing the rank of the matrix of partial derivatives of the polynomials is enough. However, by elaborating on the structure of this distinguisher, we shall explain in section 3 how it is possible to fully recover the secret key.

\subsection{Considerations on the Dimension of Vector Spaces Derived from the Public Key}

The key observation underlying the distinguisher is that, while the space of homogeneous cubic polynomials $H_{3}=H_{3,32}$ has dimension $\left(\begin{array}{c}34 \\ 3\end{array}\right)=5984$, the homogeneous cubic parts of the derivatives of the public key $\left(\partial \mathrm{PK} K_{i} / \partial x_{j}\right)_{(3)}$ actually belong to a much smaller subspace of $H_{3}$ which happens to have dimension at most 3072 . 
The case of the ASASA cryptosystem with no perturbation polynomials. As a warm-up, we first consider the encryption of the message $\left(x_{0}, \ldots, x_{31}\right)$ under the "non-perturbed" ASASA scheme, i.e. the ASASA scheme with no perturbation polynomials. We recall that all intermediate values $\left\{y_{r, i}\right\},\left\{y_{r, i}^{\prime}\right\},\left\{z_{r, i}\right\}$ and $\left\{z_{i}^{\prime}\right\}$ introduced at Section 1 can be seen as polynomials in the 32 input variables $x_{i}$ with coefficients in $k=\mathbb{F}_{16}$ (see Figure 1).

To see that the $\left(\partial \mathrm{PK}_{i} / \partial x_{j}\right)_{(3)}$, for $i=0 \ldots 127, j=0 \ldots 31$, belong to a restricted subspace, we consider the second S-box layer $S^{y}$; recall that $z_{r, i}$ denotes the $i$-th output of the S-box $S_{r}^{y}$ with input $y_{r, 0}^{\prime}, \ldots y_{r, 3}^{\prime}$. The quadratic polynomials $y_{r, i}^{\prime}$ may be written $y_{r, i}^{\prime}=\left(y_{r, i}^{\prime}\right)_{(2)}+\left(y_{r, i}^{\prime}\right)_{(1)}+\left(y_{r, i}^{\prime}\right)_{(0)}$, as the sum of a homogeneous degree 2 part, a linear part and a constant. We therefore see that the homogeneous parts of degree 4 of the polynomials $z_{r, i}$ output by the S-box $S_{r}^{y}$ are linear combinations of the terms $\left(y_{r, m}^{\prime}\right)_{(2)} \cdot\left(y_{r, n}^{\prime}\right)_{(2)}$. Let us write $\partial z_{r, i} / \partial x_{j}$ for the derivative of the output $z_{r, i}$ along the input variable $x_{j}$. There exists coefficients $a_{m, n} \in k$ such that

$$
\left(\partial z_{r, i} / \partial x_{j}\right)_{(3)}=\sum_{m, n} a_{m, n}\left(y_{r, m}^{\prime}\right)_{(2)}\left(\partial y_{r, n}^{\prime} / \partial x_{i}\right)_{(1)} .
$$

Let $Y_{(2)}^{\prime} \subset H_{32,2}$ be the vector space spanned by the 64 homogeneous quadratic parts of the polynomials $y_{r, m}^{\prime}$; it has dimension at most 64 . Let also $(D Z)_{(3)}$ be the vector space spanned by the $128 \times 32=4096$ homogeneous cubic parts of the derivatives of the polynomials $z_{r, i}$. The expression (5) above implies

$$
(D Z)_{(3)} \subset Y_{(2)}^{\prime} \cdot H_{1}
$$

The vector space $\left(D Z^{\prime}\right)_{(3)}=\left\langle\left(\partial \mathrm{PK}_{i} / \partial x_{j}\right)_{(3)}\right\rangle$ being a linear image of $(D Z)_{(3)}$ by $A^{z}$, we also have

$$
\left(D Z^{\prime}\right)_{(3)} \subset Y_{(2)}^{\prime} \cdot H_{1}
$$

and

$$
\operatorname{dim}\left(D Z^{\prime}\right)_{(3)} \leq \operatorname{dim} Y_{(2)}^{\prime} \cdot H_{1} \leq 64 \times 32=2048
$$

The general ASASA cryptosystem. For the general ASASA scheme, we have to slightly adapt the result (7) to take into account the perturbation polynomials. We refer the reader to Figure 2 for the description of ASASA used in this paragraph.

We established in our analysis of the unperturbed scheme that the homogeneous cubic parts of the derivatives of the polynomials $\left\{z_{r, i}\right\}$ belong to the vector space $Y_{(2)}^{\prime} \cdot H_{1}$, which has dimension 2048 . This still holds for the general ASASA scheme, since up to generation $z$, the perturbation polynomials do not appear. The next step in the algorithm is the linear transform $A^{z}$; its input is the concatenation $z \|\left(p_{0}, \ldots, p_{31}\right)$, where the first 96 elements are the polynomials of $z$ and the last 32 are the perturbation polynomials. This means that the polynomials of the public key $z^{\prime}$ are linear combinations of the polynomials of $z$ and the perturbation polynomials $p_{0}, \ldots p_{15}$. Let $D P$ be the vector space spanned by 
the homogeneous cubic parts $\left(\partial p_{i} / \partial x_{j}\right)_{(3)}$ of the derivatives of the perturbation polynomials; it has dimension at most $32 \times 32=1024$. We necessarily have

$$
\left(D Z^{\prime}\right)_{(3)} \subset Y_{(2)}^{\prime} \cdot H_{1}+D P .
$$

In terms of dimensions, Equation (9) implies

$$
\operatorname{dim}\left(D Z^{\prime}\right)_{(3)} \leq \operatorname{dim}\left(Y^{\prime}\right)_{(2)} \cdot H_{1}+1024 \leq 3072
$$

as claimed.

\subsection{The Distinguisher}

We now turn the observation of Section 2.1 into a working distinguisher.

Proposition 3. It is possible to distinguish the public key polynomials of the ASASA scheme from uniformly random quartic polynomials by computing the rank of a matrix of size $5984 \times 4096$ with coefficients in $\mathbb{F}_{16}$.

Proof. Let $T=\left(T_{0}, \ldots, T_{127}\right)$ be a vector of 128 polynomials that are either uniformly random quartic polynomials, or a (perturbed) ASASA public key PK. We consider the matrix $M$ of size $5984 \times 4096$ whose columns are the vectors $\left(\partial T_{i} / \partial x_{j}\right)_{(3)} \in H_{3,32}$, with the usual notations. If the $T_{i}$ are uniformly random polynomials, then the rank of $M$ is 32 times the rank of the family $\left(T_{i}\right)$; since $128 \times 32<\operatorname{dim} H_{3,32}$, according to our heuristic, this is usually $32 \times 128=4096$. If on the contrary $T$ is an ASASA public key, then by (10), the rank of $M$ is at most 3072 .

The distinguisher that returns ASASA if the rank of $M$ is $\leq 3072$, and random otherwise, succeeds with overwhelming probability. ${ }^{3}$

\section{Key Recovery}

We now present a secret key recovery attack on an ASASA scheme. The ideas used here are based on the properties already identified in Section 2, namely, the space of derivatives of PK contains information about the intermediate values between the two quadratic layers.

To attack the system, we first identify the vector space of quadratic forms manipulated in the middle of the algorithm (as output of the first S layer, and input to the second one). This is the crucial point of the cryptanalysis. It enables us to reduce the problem to two much simpler ASA problems. We then solve each ASA instance in turn. (Note that although we present a specific way to solve to

\footnotetext{
${ }^{3}$ We may also investigate the case of a reinforced ASASA scheme with more perturbation polynomials, i.e. with 96 "legitimate" outputs and $p \geq 32$ perturbations. We easily find that our distinguisher works at least for $p \leq 90$. The same bound applies to the key recovery attack of section 3 below.
} 
these two ASA instances, it is well-known that ASA instances are weak, and techniques to solve such systems can be found in the literature $[2,9,11,6]$ ).

This key-recovery only relies on linear algebra in various spaces of homogeneous polynomials. We refer the reader to Section 1.3 for some useful general results in algebra used in the attack; a few other results will be introduced on the fly when needed. For simplicity in this whole section 3, we write $Y$, instead of $Y_{(2)}^{\prime}$, for the space generated by the homogeneous quadratic parts of the polynomials of $y$.

The overall complexity of the attack is about $2^{26}$ times the computation of the rank of a square matrix of size $64 \times 96$ with coefficients in $\mathbb{F}_{16}$. We point out that our method only uses the quadratic terms of the secret quadratic layers; it is therefore also applicable to homogeneous instances of the ASASA cryptosystem.

\subsection{Computing the Middle Layer}

As already mentioned, our attack uses the same data as the distinguisher. More precisely, the key result was given at Equation (9): the vector space $D Z^{\prime}$ of derivatives of PK contains information about the space $Y \cdot H_{1}$, i.e. about the vector space $Y$ of homogeneous quadratic functions produced by the first $S$ layer. However, the observed vector space deduced from the public key also contains some unwanted vectors originating in the perturbation polynomials.

To access the space $Y \cdot H_{1}$ and see beyond the perturbation polynomials, the first step is to construct several subspaces of $D Z^{\prime}$ including $Y \cdot H_{1}$. We are then able to recover $Y \cdot H_{1}$ as the intersection of all those subspaces. In a second step we show how, from this recovered vector space, we compute the space $Y$ itself.

Eliminating the perturbations. This first steps aims at computing the vector space $Y \cdot H_{1}$ from the public key.

Recall that a public key PK of the ASASA cryptosystem is given as a vector of 128 polynomials $\mathrm{PK}_{i}$ in the 32 input variables. We define $F_{i}=\left(\mathrm{PK}_{i}\right)_{(4)}$ as the homogeneous quartic part of the public key, and $F$ as the vector space generated by all $F_{i}$.

For any derivation $\delta \in D$ and for any $f \in F$, we saw when describing the distinguisher that

$$
\delta f \in Y \cdot H_{1}+\delta P
$$

where $Y$ is the vector space generated by the 64 quadratic polynomials $\left(y_{r, i}\right)_{(2)}$, and $P$ is the vector space generated by the 32 perturbation polynomials $\left(p_{i}\right)_{(4)}$.

Let $\Delta \subset D$ be a vector space of dimension $d$. By (11), we then have

$$
\Delta F \subset Y \cdot H_{1}+\Delta P,
$$

where the right-hand space has dimension at most $64 \times 32+32 \times d=2048+32 d$. The space $\Delta F$ is generated by $128 d$ elements $\delta f_{i}$, for $\delta \in \Delta$ and $i=0, \ldots, 127$. By Proposition 2, these $128 d$ elements generate the whole space $Y \cdot H_{1}+\Delta P$ as long as $128 d \geq 2048+32 d+32$, or equivalently, $d \geq 22$. 
We now consider a family of $m$ vector spaces $\Delta_{1}, \ldots, \Delta_{m} \subset D$, each space $\Delta_{i}$ being of dimension $d=22$. We know by what precedes that for each one of them, $\Delta_{i} F=Y \cdot H_{1}+\Delta_{i} P$. This implies that

$$
\bigcap_{i=1}^{m} \Delta_{i} F=Y \cdot H_{1}+\bigcap_{i=1}^{m} \Delta_{i} P .
$$

Since $\operatorname{dim} D=32$ and $\operatorname{dim} \Delta_{i}=22$ for each $i$, the intersection of two spaces $\Delta_{i}$ generally has dimension 12 (this is always the case if we choose the $\Delta_{i}$ correctly), and likewise the intersection of three such spaces has dimension 2 , and for $m \geq 4$, we easily find $\Delta_{1}, \ldots, \Delta_{m}$ such that $\Delta_{1} \cap \ldots \cap \Delta_{m}=0$. This implies that

$$
\bigcap_{i=1}^{m} \Delta_{i} P=0 \text { for } m \geq 4 \text {. }
$$

Formula (14) means that the intersection $\bigcap_{i=1}^{m} \Delta_{i} F$ is then exactly the space $Y$. $H_{1}$ :

$$
\bigcap_{i=1}^{m} \Delta_{i} F=Y \cdot H_{1} \text { for } m \geq 4 .
$$

Computing the middle terms. This part explains how we recover the 64dimensional space $Y$ from the space $Y \cdot H_{1} \subset H_{3}$ obtained during the previous step.

We first prove a short lemma. Let $V \subset H_{2}$ be a vector space of dimension $d$ and basis $\left(v_{j}\right)$. The vector space $V \cdot H_{1} \subset H_{3}$ is generated by the $32 d$ elements $x_{i} v_{j}$. If $d \leq 186$, then $32 d \leq \operatorname{dim} H_{3}-32$; by Proposition 2, we therefore expect these $32 d$ elements to be linearly independent in $H_{3}$. This implies that $\operatorname{dim}\left(V \cdot H_{1}\right)=32 d$. In particular, this means that when $V$ has dimension $\leq 186$, the correspondence between $(\operatorname{dim} V)$ and $\left(\operatorname{dim} V \cdot H_{1}\right)$ behaves, with very high probability, as a strictly increasing function.

We now use this lemma to characterize the space $Y$. Let $\bar{Y} \subset H_{2}$ be the vector space of all functions $g$ such that, for all $i, g x_{i} \in Y \cdot H_{1}$. Trivially, $Y \subset \bar{Y}$ and $\bar{Y} \cdot H_{1}=Y \cdot H_{1}$. Since $\operatorname{dim} Y=64 \leq 186$, we are in the conditions of the previous lemma, which implies that $Y=\bar{Y}$ with overwhelming probability.

We may easily compute the space $\bar{Y}$ from $Y \cdot H_{1}$ as follows. For each $i=$ $0, \ldots, 31$, we define $G_{i}$ as the subspace of multiples of $x_{i}$ in $Y \cdot H_{1}$; by definition, $\bar{Y}$ is the intersection of all spaces $x_{i}^{-1} G_{i}$,

$$
\bar{Y}=\bigcap_{i=0}^{31} x_{i}^{-1} G_{i} .
$$

\subsection{Solving a Quadratic ASA Layer}

As already mentioned, there exists generic techniques $[2,9,11,6]$ for inverting a public key in the ASA form. We give our own solution here, as it is simple and 
works well in the particular case of an ASA layer based on quadratic S-boxes. We shall use this technique twice, once for the inner ASA layer, and then once more for the outer one.

This section and the next one (Section 3.3) are mutually independent. We present the inner layer ASA first since it is easier to explain.

Notations. The inner ASA layer is represented as the (known) vector space $Y$ generated by the 64 (unknown) quadratic forms $y_{r, i}$ in the 32 input variables $x_{i}$. We restrict ourselves here to the homogeneous part of the $y_{r, i}$, since this case is more difficult to solve, but easier to present.

For each fixed $r$, the eight functions $y_{r, 0}, \ldots, y_{r, 7}$ are quadratic forms in the same 4 fixed linear combinations $x_{r, 0}^{\prime}, \ldots, x_{r, 3}^{\prime}$ of the input variables $x_{i}$. We write $X_{r}$ for the vector space generated by the $x_{r, i}^{\prime}$. We may also decompose the space of differentials $D=\left\langle\partial / \partial x_{i}\right\rangle$ according to the S-boxes; namely, for each $r$, we define $D^{r}$ as the set of all elements $\delta \in D$ whose restriction to $X_{r}$ is zero.

We note that $\operatorname{dim} X_{r}=4$ and $\operatorname{dim} D^{r}=28$. Therefore, for a given $r$, an uniformly random element of $D$ belongs to $D^{r}$ with probability $q^{-4}=2^{-16}$.

Separating the inputs of the S-boxes. We show that we are able to identify the elements of $D^{r}$, i.e. the differentials that vanish on the inputs of a particular S-box. ${ }^{4}$

For any quadratic form $f \in Y$ and any $\delta \in D$, the function $\delta f$ is a linear form of $x \in X$; this means that $(\delta f)(x)$ is a trilinear function of $(\delta, f, x)$. Therefore, for a fixed value of $\delta$, it is a bilinear function of $(f, x)$. We write $F_{\delta}$ for this bilinear form. It is represented by a matrix of size $64 \times 32$ whose coefficients are the $\left(\delta f_{i}\right)\left(x_{j}\right)$, where $\left(f_{i}\right)$ is a basis of $Y$ and $\left(x_{j}\right)$ is the standard basis of $X$.

Let $f \in Y$; we can write $f$ as a sum $\sum f_{s}$ for $s=0, \ldots, 7$, where $f_{s}$ is a quadratic form on $X_{s}$. For any $\delta \in D^{r}$, we have $\delta f_{r}=0$, so that $\delta f$ is the sum of the $\delta f_{s}$ for $s \neq r$. Since each one of these terms uses only the variables from $X_{s}$, this means that $(\delta f)(x)=0$ for $x \in X_{r}$. Put differently, the kernel of $F_{\delta}$ (here seen as a linear map from $X$ to the dual of $Y$ ) contains $X_{r}$.

Now let $\delta$ be an element of $D$ not belonging to any of the $D^{r}$ : since the maps $\left(\delta f_{i}\right)$ are 64 random linear forms on the 32 -dimensional space $X$; by Proposition 2, with overwhelming probability, the intersection of their kernels is zero.

As a result, we see that the rank of the matrix $F_{\delta}$ is always $\leq 28$ if $\delta$ belongs to at least one of the $D^{r}$ and 32 with overwhelming probability otherwise. This also provides a test, given two elements $\delta$ and $\varepsilon$ of $D^{r}$ and $D^{s}$, for the equality $r=s$ : since the kernels of the matrices $F_{\delta}, F_{\varepsilon}$ contain respectively $X_{r}$ and $X_{s}$, their intersection is non-trivial when $r=s$.

\footnotetext{
${ }^{4}$ Since the elements of $Y$ are quadratic forms, their differentials are exactly the associated polar forms. This means that we may represent the derivations as elements of $X$, using the relation $(\delta f)(x)=f(x+\delta)-f(x)-f(\delta)$; in this view, the space $D^{r}$ is the direct sum of all the $X_{s}$ for $s \neq r$. However, we chose to use an explanation based on differentials, since this is both closer to the differential cryptanalysis point of view, and easier to generalize to polynomials of higher degree.
} 
The algorithm. We compute the spaces $X_{r}$ and $D^{r}$ at the same time, and up to a permutation of $\{0, \ldots, 7\}$, since we do not know the labeling of the S-boxes. For each $r=0, \ldots, 7$, we keep candidates $U_{r}$ and $V_{r}$ for the spaces $X_{r}$ and $D^{r}$; initially, $U_{r}$ is the whole space $X$ while the space $V_{r}$ is empty. During the whole algorithm, we have $U_{r} \supset X_{r}$ and $V_{r} \subset D^{r}$, with both inclusions being equalities at the end. We also note that, at every step of the alorithm, $U_{r}$ is the intersection of the kernels of all elements of $V_{r}$.

We now describe the algorithm. We randomly generate elements $\delta$ of $D$ and compute the kernel $K \subset X$ of the matrix $F_{\delta}$. If this kernel has dimension at least 4, then it intersects non-trivially one of the spaces $U_{r}$ and the intersection also has dimension at least 4 . We then update $V_{r}$ to $V_{r} \oplus\langle\delta\rangle$ and $U_{r}$ to $U_{r} \cap K$. The algorithm ends when each space $V_{r}$ has the required dimension 28, as then $V_{r}=$ $D^{r}$ as required, and therefore $U_{r}=X_{r}$.

Recovering the S-boxes. Once the spaces $X_{r}$ are known, computing the S-boxes is easy. We write $Y_{r}$ for the vector space generated by the 8 outputs of the S-box $S_{r}^{x}$, and $X^{r}$ for the direct sum of all $X_{s}$ for $s \neq r$. Then $Y_{r}$ is exactly the set of elements of $Y$ that vanish on all points of $X^{r}$. We may therefore compute $Y_{r}$ with linear algebra. (Another possibility is to use the derivations spaces $D^{r}$, also computed in the previous step, since $Y_{r}$ is the set of elements $f \in Y$ such that, for all $\left.\delta \in D^{r}, \delta f=0\right)$. Once bases of both $X_{r}$ and $Y_{r}$ are known, we recover the secret functions $S_{r}^{x}$ by interpolation.

Complexity. Since we need to generate on average $q^{4} \times 28 \times 8 \approx 15 \cdot 10^{6}$ elements of $X$, the expected cost for the execution of this algorithm is approximately $2^{23.8}$ times that of the computation of the kernel of a matrix of size $32 \times 64$ with entries in $\mathbb{F}_{16}$.

\subsection{Solving the Outer ASA Layer}

We again use the representation of the middle layer as a 64-dimensional vector space $Y$ of quadratic forms computed in 3.1. We now determine the output functions $F_{i}$ as linear combinations of quadratic forms in the elements of $Y$ and the 32 perturbation polynomials $p_{i}$.

Computing the outputs of the S-boxes. We recall that $F$ is the vector space generated by the homogeneous quartic part of the ASASA public key. This vector space is the direct sum of the 32 -dimensional space $P$ generated by the homogeneous quartic parts of the perturbations, and the 96-dimensional vector space $Z$ generated by the outputs of the 16 S-boxes $S_{r}^{y}$. Since the middle layer $Y$ is known as a result of Section 3.1, we may compute $Z$ as the intersection $Z=(Y \cdot Y) \cap F$. 
Reducing to a quadratic ASA layer. We now study the 16 S-boxes $S_{r}^{y}$. We already know the 64-dimensional vector space $Y$ of their (quadratic) inputs and the 96-dimensional vector space $Z$ of their (quartic) outputs. Our goal for now is to rewrite each element of $Z$ as an explicit quadratic function of the elements of $Y$, so as to be able to apply the techniques of Section $3.2 .^{5}$

We represent $Y$ by its Hermite normal basis relative to a particular basis of $H_{2}$ : the first 32 elements of $Y$ have the form $u_{i}=x_{i}^{2}+\ldots$ for $i=1, \ldots, 32$, the next 31 elements have the form $v_{i}=x_{1} x_{i}+\ldots$ for $i=2, \ldots, 32$, and the last one is $w=x_{2} x_{3}+\ldots$, where none of the omitted "..." expressions contain either squares or terms $x_{1} x_{i}$ or $x_{2} x_{3}$. In the (unlikely) case where the Hermite normal form of $Y$ does not contain the monomials $x_{1}^{2}, \ldots, x_{2} x_{3}$, we may always replace the public key $F$ by its composition $F \circ \sigma$ by a random invertible linear transformation of the input variables, such that $Y \circ \sigma$ has the suitable Hermite normal form.

We now consider a basis of the space $Z$. Any term of the form $\lambda x_{i}^{4}$ appearing in a basis element of $Z$ comes, in its expression as a quadratic forms over $Y$, from a term $\lambda u_{i}^{2}$. Likewise, any term of the form $\mu x_{1}^{3} x_{i}$ comes from a term $\mu u_{1} v_{i}$, and so on.

In this way, we identify the second ASA layer as a quadratic map from $Y$ to $Z$.

Solving the ASA problem. The problem we have to solve is now almost identical to the one we solved in Section 3.2, except that we now have 16 instead of 8 S-boxes, and 96 instead of 64 quadratic forms.

Applying the previous algorithm to this case thus has a global complexity of approximately $q^{4} \times 60 \times 16 \approx 2^{25.9}$ times the cost of computing the kernel of a matrix of size $64 \times 96$ with entries in $\mathbb{F}_{16}$. This is the dominant step in the key recovery procedure. We estimate the corresponding computational cost to a few CPU-hours.

\subsection{Computing the Inhomogeneous Terms}

We just presented an algorithm computing the homogeneous part (of degree two) of the quadratic S layers of the ASASA public key. These homogeneous terms represent the largest part of the secret key. Once they are computed, recovering the inhomogeneous terms (of degree one) is quite simple.

Each output S-box has one such linear term, represented by four coefficients; in total, there are therefore $(64+96) \times 4=640$ unknown coefficients. We consider the homogeneous parts of degree one and three of the public key $\mathrm{PK}_{i}$. These

\footnotetext{
${ }^{5}$ We note that in the (very unlikely) case where the computation of the space $Y$ performed in Section 3.1 returned a space $Y^{\prime} \supsetneq Y$, the computation performed here will allow us to remove the few extra elements of $Y^{\prime}$ : namely, since the elements of $Z$ are quadratic forms in the elements of $Y$, the unneeded elements of $Y^{\prime}$ will not appear in these expressions. This means that, in practice, this step (Section 3.3) should be performed before the inner step (Section 3.2).
} 
functions are linear in the unknown inhomogeneous terms. Since there are exactly $\left(\operatorname{dim} H_{1}+\operatorname{dim} H_{3}\right) \times 32=192512$ such functions, we have enough linear equations to recover all inhomogeneous terms.

\section{Conclusion}

We presented a very efficient distinguisher on the main ASASA scheme proposition of [3], that evolved into a full key-recovery algorithm with very reasonable complexity. The complexity of the attack can be approximated by the cost of computing the kernels of $2^{26}$ matrices of size $64 \times 96$ with entries in $\mathbb{F}_{16}$. This cost is well within practical limits. A classical venture to "repairing" a multivariate cryptosystem is to consider the homogeneous variant of the broken scheme. We point out that our cryptanalysis works by considering the homogenous quartic parts of the polynomials of the public key, thus defeats any such attempt. Another possibility would be to reinforce the scheme by adding more perturbation polynomials. However, our attack still works without any modification even for a larger number of perturbations.

\section{References}

1. Eli Biham. Cryptanalysis of Patarin's 2-round public key system with $\mathrm{S}$ boxes (2R). In Advances in Cryptology-EUROCRYPT 2000, pages 408-416. Springer, 2000 .

2. Olivier Billet, Henri Gilbert, and Charaf Ech-Chatbi. Cryptanalysis of a white box AES implementation. In Selected Areas in Cryptography 2005, pages 227-240. Springer, 2005.

3. Alex Biryukov, Charles Bouillaguet, and Dmitry Khovratovich. Cryptographic schemes based on the ASASA structure: Black-box, white-box, and public-key. In Advances in Cryptology-ASIACRYPT 2014, pages 63-84. Springer, 2014.

4. Alex Biryukov and Adi Shamir. Structural cryptanalysis of SASAS. In Advances in Cryptology-EUROCRYPT 2001, pages 395-405. Springer, 2001.

5. Joan Daemen. Cipher and hash function design strategies based on linear and differential cryptanalysis. $\mathrm{PhD}$ thesis, Doctoral Dissertation, March 1995, KU Leuven, 1995.

6. Yoni De Mulder, Peter Roelse, and Bart Preneel. Cryptanalysis of the Xiao-Lai White-Box AES Implementation. In Selected Areas in Cryptography 2013, pages 34-49. Springer, 2013.

7. Ye Ding-Feng, Lam Kwok-Yan, and Dai Zong-Duo. Cryptanalysis of "2R" schemes. In Advances in Cryptology-Crypto'99, pages 315-325. Springer, 1999.

8. Jean-Charles Faugère and Ludovic Perret. Cryptanalysis of $2 R^{-}$schemes. In Advances in Cryptology-CRYPTO 2006, pages 357-372. Springer, 2006.

9. Louis Goubin, Jean-Michel Masereel, and Michaël Quisquater. Cryptanalysis of white box DES implementations. In Selected Areas in Cryptography 2007, pages 278-295. Springer, 2007.

10. Tsutomu Matsumoto and Hideki Imai. Public quadratic polynomial-tuples for efficient signature-verification and message-encryption. In Advances in Cryptology - EUROCRYPT'88, pages 419-453. Springer, 1988. 
11. Wil Michiels, Paul Gorissen, and Henk DL Hollmann. Cryptanalysis of a generic class of white-box implementations. In Selected Areas in Cryptography 2009, pages 414-428. Springer, 2009.

12. Morris Newman. Integral matrices. Academic Press, 1972.

13. Jacques Patarin. Cryptanalysis of the Matsumoto and Imai public key scheme of Eurocrypt'88. In Advances in Cryptology-CRYPT0'95, pages 248-261. Springer, 1995.

14. Jacques Patarin. Asymmetric cryptography with a hidden monomial. In Advances in Cryptology-CRYPTO'96, pages 45-60. Springer, 1996.

15. Jacques Patarin and Louis Goubin. Asymmetric cryptography with S-Boxes Is it easier than expected to design efficient asymmetric cryptosystems? Information and Communications Security, pages 369-380, 1997.

16. Vincent Rijmen and Bart Preneel. A family of trapdoor ciphers. In Fast Software Encryption, pages 139-148. Springer, 1997.

17. Bernd Sturmfels. Algorithms in invariant theory. Springer Science \& Business Media, 2008.

18. Hongjun Wu, Feng Bao, Robert H Deng, and Qin-Zhong Ye. Cryptanalysis of Rijmen-Preneel trapdoor ciphers. In Advances in Cryptology-Asiacrypt'98, pages 126-132. Springer, 1998. 